\title{
Una Propuesta para el Análisis de las Prácticas Matemáticas de Futuros Profesores sobre Derivadas
}

\author{
A Proposal for the Analysis of Prospective Teachers' Mathematical \\ Practices on Derivatives
}

\author{
Luis R. Pino-Fan* \\ Juan D. Godino** \\ Vicenç Font $^{* * *}$
}

\begin{abstract}
Resumen
En el presente trabajo se informa de los resultados obtenidos al analizar las prácticas matemáticas de una muestra de futuros profesores de secundaria/bachillerato a propósito de dos tareas sobre derivadas. Este análisis ejemplifica el uso y alcances de las nociones prácticas matemáticas y configuración de objetos y procesos, proporcionadas por el Enfoque Onto-Semiótico (EOS) del conocimiento matemático, para la caracterización de los conocimientos del profesorado de matemáticas. Los resultados del análisis evidencian tanto una desconexión entre los distintos significados parciales de la derivada como la necesidad de potenciar el conocimiento del contenido. Este aprendizaje puede hacerse mediante actividades que favorezcan el uso e identificación de objetos matemáticos, sus significados y los procesos involucrados en la solución de tareas matemáticas.
\end{abstract}

Palabras clave: Formación de Profesores. Conocimiento del Profesor. Enfoque Ontosemiótico. Prácticas matemáticas. Derivada.

\begin{abstract}
In this paper, we present results obtained from the analysis of prospective high school teachers' mathematical practices about two tasks on derivatives. This analysis exemplifies the use and scopes of the notions of mathematical practices and configurations of objects and processes, provided by the Onto-Semiotic Approach (OSA) of mathematical knowledge, to characterize the knowledge of mathematics teachers. The analysis of the students' responses show both a disconnection between the different partial meanings of the derivative as well as the need to enhance the content knowledge through activities that encourage the use and identification of mathematical objects, their meanings, and the processes involved in the solution of mathematical tasks.
\end{abstract}

Keywords: Teacher's Education. Teacher's Knowledge. Onto-Semiotic Approach. Mathematical Practices. Derivative.

\footnotetext{
* Doctor en Didáctica de la Matemática por la Universidad de Granada (UGR). Profesor del Departamento de Ciencias Exactas de la Universidad de Los Lagos (ULAGOS), Santiago, Chile. Dirección postal: Av. República, 517, Barrio República, 8370341, Santiago, Chile. E-mail: luis.pino@ulagos.cl

** Doctor en Matemáticas por la Universidad de Granada (UGR). Profesor Catedrático de Didáctica de la Matemática de la Universidad de Granada (UGR), Granada, España. Dirección postal: Facultad de Educación, Campus Cartuja, 18071, Granada, España. E-mail: jgodino@ugr.es

**** Doctor en Filosofía y Ciencias de la Educación por la Universidad de Barcelona (UAB). Profesor Titular del Departamento de Didáctica de las Ciencias Experimentales y de la Matemática de la Universidad de Barcelona (UAB), Barcelona, España. Dirección postal: Calle Figols, 15, 08028, Barcelona, España. E-mail: vfont@ub.edu
} 


\section{Problema y antecedentes}

Las características de la comprensión del concepto de derivada de los estudiantes de Bachillerato y primeros cursos de universidad es un tema que ha sido ampliamente investigado por diversos autores, desde distintas aproximaciones teóricas - particularmente las cuestiones de índole cognitiva e instruccional - (ASIALA et al., 1997; BAKER; COOLEY; TRIGUEROS, 2000; SÁNCHEZ-MATAMOROS et al., 2006; ARTIGUE; BATANERO; KENT, 2007; GARCÍA; LLINARES; SÁNCHEZ-MATAMOROS, 2011). No obstante, aunque también ha sido de interés caracterizar los conocimientos de los profesores para una enseñanza efectiva de la derivada (GAVILÁN; GARCÍA; LLINARES, 2007; BADILLO; AZCÁRATE; FONT, 2011; SÁNCHEZ-MATAMOROS et al., 2012), pocos han sido los estudios centrados en los profesores y su relación con la gestión de los aprendizajes sobre derivada. Algunos de estos trabajos han centrado la atención en profesores en ejercicio y otros en estudiantes para profesor de matemáticas, para interpretar la naturaleza del concepto de derivada, así como del conocimiento y comprensión del mismo.

En este artículo presentamos resultados parciales, pero relevantes, de una investigación más amplia (PINO-FAN, 2013) centrada en la caracterización de los Conocimientos Didáctico-Matemáticos $(\mathrm{CDM})$ de futuros profesores de matemáticas de bachillerato. Se interpreta el CDM en el sentido propuesto en Godino (2009) quien, aplicando el enfoque onto-semiótico (EOS) del conocimiento y la instrucción matemática (GODINO; BATANERO; FONT, 2007), interpreta y amplía las categorías de análisis de los conocimientos del profesor de matemáticas propuestas por otros autores (SHULMAN, 1986; BALL, 2000; ROWLAND; HUCKSTEP; THWAITES, 2005; HILL; BALL; SCHILLING, 2008; SCHOENFELD; KILPATRICK, 2008). Concretamente, en este artículo nos proponemos desarrollar los siguientes objetivos:

1. Ejemplificar el uso de una de las categorías de análisis del CDM, para el caso de los conocimientos de futuros profesores de Bachillerato sobre la derivada. Para poder realizar esta ejemplificación se ha diseñado y aplicado un cuestionario a una muestra de 53 futuros profesores de una universidad mexicana. Dado que no es posible incluir en un cuestionario ítems que contemplen las diferentes facetas e indicadores del CDM, esta investigación se centra en aspectos relevantes de la faceta epistémica de dicho conocimiento didáctico-matemático. 
2. Ejemplificar el uso de las herramientas teórico-metodológicas, sistemas de prácticas y configuración de objetos y procesos que nos proporciona el Enfoque OntoSemiótico (EOS) para analizar los conocimientos puestos en juego en la solución de tareas matemáticas que involucran el uso del objeto derivada.

3. Analizar, describir y caracterizar, mediante la identificación de tipologías de configuraciones cognitivas, los conocimientos de la muestra de futuros profesores, a propósito de dos de las tareas del cuestionario que se han seleccionado para su discusión en este artículo. Los tipos de configuraciones cognitivas identificadas constituyen un desglose analítico del conocimiento de los futuros profesores sobre la derivada. Con base en esta caracterización de conocimientos se ejemplificarán el nivel y las herramientas de análisis del CDM descritos en los objetivos anteriores.

Una vez presentados los objetivos y los antecedentes de la investigación, en la siguiente sección describimos las nociones del marco teórico que se usan en el artículo y la metodología. En la sección 3 describimos las configuraciones de objetos y procesos manifestadas por la muestra de futuros profesores, a propósito de la resolución de dos de las tareas del cuestionario construido. Contemplamos el análisis relativo a dos de las tareas básicamente por dos motivos: 1) motivos de espacio; y 2) la complejidad de los protocolos de resolución de dichas tareas, permite mostrar el uso y potencialidad de las herramientas teóricas que se contemplan en los dos objetivos de esta investigación. En la sección 4 presentamos resultados globales, para dichas tareas, referidos a la incidencia de los tipos de configuraciones cognitivas identificadas y sobre el grado de corrección de las respuestas. Finalmente, interpretamos los resultados en términos de las necesarias relaciones entre los tipos de conocimientos del profesor de matemáticas, restringido a la faceta epistémica del CDM.

\section{Marco teórico y metodología}

El análisis didáctico de los procesos de enseñanza y aprendizaje de contenidos matemáticos se realiza, en el EOS, distinguiendo en los mismos seis facetas o dimensiones: epistémica, cognitiva, afectiva, interaccional, mediacional y ecológica (GODINO; BATANERO; FONT, 2007). Para cada faceta se distinguen distintas componentes y se han desarrollado diversas herramientas que permiten su análisis. En este trabajo tenemos en cuenta las facetas epistémica y cognitiva del análisis didáctico basado en el EOS. La faceta 
epistémica de un proceso de estudio matemático refiere a los significados institucionales puestos en juego en cada una de las fases de dicho proceso (preliminar, diseño, implementación y evaluación). Tales significados son interpretados en términos de sistemas de prácticas y configuraciones de objetos y procesos. Mientras que la faceta cognitiva refiere a los significados personales - de los estudiantes o, en nuestro caso, de los futuros profesores - descritos en los distintos momentos de su desarrollo en términos de sistemas de prácticas personales y configuraciones cognitivas de objetos y procesos.

Estas herramientas de análisis didáctico han sido utilizadas para elaborar un sistema de categorías de los conocimientos del profesor de matemáticas que se designa como modelo del Conocimiento Didáctico-Matemático $(\mathrm{CDM})$ de los profesores. Para nuestra investigación hemos adoptado este modelo (CDM), el cual ha sido planteado por Godino (2009) y refinado en diversas publicaciones (GODINO; PINO-FAN, 2013; PINO-FAN; FONT; GODINO, 2014; PINO-FAN; GODINO; FONT, 2013), al seno del EOS. El modelo CDM propone tres dimensiones del conocimiento de los profesores: 1) matemática; 2) didáctica; 3) meta didáctico-matemática. La dimensión matemática incluye las categorías de conocimiento común del contenido -el cual es entendido como el conocimiento matemático que es suficiente para resolver tareas propias del nivel educativo en el cual se está inmerso en un momento concreto, por ejemplo, bachillerato- y conocimiento ampliado del contenido conocimiento matemático que posibilita al profesor realizar conexiones con contenidos que están más adelante en el currículum de matemáticas y orientar a los estudiantes al estudio de contenidos posteriores. La dimensión didáctica incluye las categorías o facetas de conocimiento: epistémica (conocimiento especializado de la dimensión matemática), cognitiva (conocimientos sobre los estudiantes), afectiva (conocimiento sobre las creencias, concepciones y actitudes de los estudiantes), mediacional (conocimientos sobre los recursos y medios que influyen en los aprendizajes de los estudiantes), interaccional (conocimientos sobre el tipo de interacciones que favorecen el aprendizaje) y ecológica (conocimientos sobre el currículo y su relación con el contexto social, político y económico). La dimensión meta didáctico-matemática refiere a los conocimientos necesarios para reflexionar sobre la propia práctica y valorar la idoneidad didáctica (GODINO, 2013) de los procesos de enseñanza y aprendizaje de las matemáticas.

Cuando el foco de atención son los conocimientos que el profesor de matemáticas debe poner en juego como organizador y gestor, en los diversos momentos de un proceso de enseñanza y aprendizaje, tales conocimientos incluyen los relativos a cada una de las 
dimensiones y de las seis facetas implicadas en tales procesos. Así, cuando se habla de la faceta epistémica del CDM se refiere al conocimiento que tiene o debe tener el profesor sobre el contenido matemático como objeto institucional cuya enseñanza se planifica, implementa y evalúa (PINO-FAN; GODINO; FONT, 2013).

Esta modelización de los conocimientos del profesor de matemáticas ha sido utilizada para el diseño de un cuestionario cuyo objetivo es la evaluación de los conocimientos de una muestra de futuros profesores de Bachillerato sobre la derivada, restringida tal evaluación a aspectos relevantes de la faceta epistémica de dichos conocimientos (PINO-FAN, 2013; PINO-FAN; GODINO; FONT, 2013). En dichos estudios se sugieren que las nociones prácticas matemáticas y configuración de objetos y procesos, propuestas al seno del EOS, son herramientas teóricas y metodológicas que permiten la exploración, análisis y caracterización de los conocimientos relativos a la faceta epistémica del CDM.

La noción prácticas matemáticas refiere a la descripción de las acciones realizadas para resolver las tareas matemáticas propuestas para contextualizar los contenidos y promover el aprendizaje. También se describen las líneas generales de actuación del docente, y de los discentes cuando es necesario. Mientras que la noción configuración de objetos y procesos refiere a la descripción de objetos (situaciones problemas, elementos linguísticos, conceptos/definiciones, proposiciones/propiedades, procedimientos y argumentos) y procesos matemáticos que intervienen en la realización de las prácticas, así como los que emergen de ellas. La finalidad de este nivel es describir la complejidad de objetos matemáticos (FONT; GODINO; GALLARDO, 2013) y significados de las prácticas matemáticas como factor explicativo de los conflictos en su realización y de la progresión del aprendizaje. En la sección 3, tal y como lo señalamos anteriormente, se realiza la puesta en marcha de estas dos nociones para el análisis, descripción y caracterización de las prácticas matemáticas realizadas por una muestra de profesores en formación inicial, a propósito de la solución de dos tareas sobre derivadas.

No debemos dejar de lado que nuestra investigación se inscribe dentro de un enfoque metodológico de tipo mixto (JOHNSON; ONWUEGBUZIE, 2004), puesto que se trata de un estudio de tipo exploratorio en el que se considera la observación de variables cuantitativas (grado de corrección de los ítems: respuestas correctas, parcialmente correctas e incorrectas) y cualitativas (tipo de configuración cognitiva movilizada como parte de las prácticas matemáticas de los futuros profesores). 
Para el estudio cualitativo nos apoyamos en la técnica denominada análisis ontosemiótico (GODINO, 2002), la cual permite describir sistemáticamente tanto la actividad matemática realizada por los futuros profesores al resolver problemas (práctica matemática), como los objetos matemáticos primarios (elementos linguísticos, conceptos, proposiciones, procedimientos y argumentos) y procesos que intervienen en las prácticas que permiten su resolución.

\subsection{Sujetos y contexto}

El cuestionario, y por ende las dos tareas que analizamos en este documento, se aplicó a una muestra de 53 estudiantes de los últimos cursos (sexto y octavo semestre) de la Licenciatura en Enseñanza de las Matemáticas que se imparte en la Facultad de Matemáticas de la Universidad Autónoma de Yucatán en México. Dicha licenciatura tiene una duración de cuatro años (8 semestres). Cabe señalar que los estudiantes en nuestro estudio, futuros egresados de la licenciatura en enseñanza de las matemáticas, suelen trabajar como profesores en los niveles de bachillerato o universitario en el estado de Yucatán en México. Los 53 estudiantes a los que se les aplicó el cuestionario habían cursado cálculo diferencial en el primer semestre de la licenciatura y, a lo largo de ella, tomaron otros cursos relacionados con el análisis matemático (cálculo integral, cálculo vectorial, ecuaciones diferenciales etc.). También habían cursado materias relacionadas con las matemáticas y su didáctica.

Cabe señalar que el cuestionario diseñado incluye nueve tareas centradas, fundamentalmente, en la evaluación de la dimensión matemática y aspectos parciales de la faceta epistémica del conocimiento didáctico-matemático (CDM). Dichos aspectos parciales, según lo planteado en Pino-Fan, Godino y Font (2013), están relacionados con los conocimientos del contenido matemático. De esta forma, las tareas incluidas en el cuestionario responden básicamente a dos características. Primeramente, consideramos que las tareas deben proporcionar información sobre el grado de ajuste del significado personal de los futuros profesores respecto del significado global u holístico del objeto derivada (PINOFAN; FONT; GODINO, 2011). Para lograrlo, se incluyeron ítems que activan distintos sentidos para el objeto derivada (pendiente de la recta tangente, razón instantánea de cambio y tasa instantánea de variación). La segunda característica de los ítems seleccionados es que responden a los diferentes tipos de representaciones activados en los tres subprocesos, que según Font (1999), intervienen en el cálculo de la función derivada: a) traducciones y 
conversiones entre las distintas formas de representar $f(x)$; b) el paso de una forma de representación de $f(x)$ a una forma de representación de $f^{\prime}(x) ; \mathrm{y}$ c) traducciones y conversiones entre las distintas formas de representar $f^{\prime}(x)$.

Así, podemos decir que el cuestionario contempló tres tipos de tareas, todas relacionadas con el contenido matemático (PINO-FAN; FONT; GODINO, 2014): 1) aquellas que piden poner en juego el conocimiento común del contenido (este conocimiento hace referencia a la resolución de tareas matemáticas que se proponen usualmente en el currículo del bachillerato); 2) aquellas que requieren del conocimiento ampliado del contenido (generalizar tareas sobre conocimiento común y/o realizar conexiones con objetos matemáticos más avanzados en el currículo de bachillerato); 3) aquellas que requieren de aspectos parciales de la faceta epistémica - conocimiento especializado del contenido - (usar distintas representaciones, distintos significados parciales de un objeto matemático, resolver el problema mediante diversos procedimientos, dar diversas argumentaciones válidas, identificar los conocimientos puestos en juego durante la resolución de una tarea matemática etc.). Las tareas que hemos seleccionado para discutir en este artículo, y que presentamos en la siguiente sección, pertenecen a los grupos 3 y 2, respectivamente.

\subsection{Las tareas}

A continuación se presentan dos de las tareas incluidas en el cuestionario diseñado por Pino-Fan (2013), que hemos seleccionado para su discusión en este trabajo. Análisis epistémicos previos a la implementación de estas dos tareas pueden encontrarse en Pino-Fan, Godino y Font (2013). No obstante, el análisis y descripción de las configuraciones cognitivas que resultan de la implementación de estas dos tareas, y la ejemplificación del uso de las herramientas teóricas y metodológicas descritas para la realización de dichos análisis, son el objeto central de este estudio.

Primeramente, la Tarea 4 (Figura 1), utilizada en el trabajo de Viholainen (2008), indaga sobre el conocimiento especializado del contenido - o faceta epistémica del CDM (PINO-FAN; GODINO; FONT, 2013) de los futuros profesores, puesto que requiere, para su resolución, el empleo de diversas representaciones (gráfica, descripción verbal, fórmula) y diversas justificaciones para la proposición la derivada de una función constante siempre es igual a cero. En estas justificaciones se pueden movilizar distintas interpretaciones de la 
derivada: pendiente de la recta tangente, razón instantánea de cambio y tasa instantánea de variación.

$$
\begin{aligned}
& \text { Tarea } 4 \\
& \text { a) Explica, mediante el uso de representaciones gráficas, por qué la derivada de una función } \\
& \text { constante siempre es igual a cero. } \\
& \text { b) Usando la definición formal de la derivada, prueba que la derivada de una función constante } \\
& \text { es cero. }
\end{aligned}
$$

Figura 1 - Tarea 4 del cuestionario CDM-derivada

La Tarea 8 (Figura 2) proporciona información sobre el conocimiento ampliado de los profesores, ya que se trata de una aproximación a la derivada de una función (descrita por los valores de la tabla) en el punto $t=0,4$ a través de valores numéricos de dicha función. Además, la tarea 8 no es un problema escolar típico del nivel bachillerato, y requiere la comprensión del objeto derivada por parte de los futuros profesores, al menos en su acepción como razón instantánea de cambio, y concretamente, la derivada en un punto como velocidad instantánea. La solución de esta tarea se puede realizar mediante diferentes métodos, por ejemplo, la interpolación polinómica de Lagrange, lo cual sustenta la categorización de esta tarea como evaluadora del conocimiento ampliado.

Algunos lectores podrán encontrar que el planteamiento que se hace de la tarea es incorrecto, puesto que no responde al modelo matemático que regula la caída de graves, en este caso desplazamiento retardado, modelo que permitiría encontrar fácilmente una representación simbólica de la función que describe la distancia. Por lo tanto, podría parecer que abordar la tarea mediante interpolación polinómica de Lagrange carece de sentido. No obstante, el objetivo de plantear la tarea tal como lo hacemos en este documento, es precisamente que los datos no se ajusten de manera sencilla a un modelo físico-matemático conocido, y así los profesores demuestren su 'comprensión' en torno al objeto matemático que se está movilizando.

\section{Tarea 8}

Una pelota se lanza al aire desde un puente de 11 metros de altura. $f(t)$ denota la distancia a la que se encuentra la pelota del suelo en un tiempo $t$. Algunos valores de $f(t)$ se recogen en la siguiente tabla:

\begin{tabular}{lrrrrrrr}
\hline$t($ sec. $)$ & 0 & 0.1 & 0.2 & 0.3 & 0.4 & 0.5 & 0.6 \\
$f(t)(\mathrm{m})$. & 11 & 12.4 & 13.8 & 15.1 & 16.3 & 17.4 & 18.4 \\
\hline
\end{tabular}

De acuerdo con la tabla, ¿cuál es la velocidad de la pelota cuando alcanza una altura en $t=$ 0.4 segundos? Justifica la elección de tu respuesta.
a) $11.5 \mathrm{~m} / \mathrm{s}$
b) $1.23 \mathrm{~m} / \mathrm{s}$
c) $14.91 \mathrm{~m} / \mathrm{s}$
d) $16.3 \mathrm{~m} / \mathrm{s}$
e) Otro

Figura 2 - Tarea 8 del cuestionario CDM-derivada 


\section{Análisis de las configuraciones cognitivas asociadas a la resolución de las tareas 4 y 8}

Para el análisis cualitativo de las respuestas de los estudiantes, utilizamos algunas herramientas del enfoque ontosemiótico descritas en el apartado 2, en particular, aquellas que nos ayudan a analizar sistemáticamente las prácticas matemáticas desarrolladas por los futuros profesores para resolver los problemas propuestos, así como los objetos primarios (elementos lingüísticos, conceptos, proposiciones, procedimientos y argumentos) y procesos (particularización - generalización, materialización - idealización) implicados en la realización de dichas prácticas (GODINO et al., 2011).

\subsection{Configuraciones cognitivas asociadas a la resolución de la tarea 4}

En las resoluciones que dieron los futuros profesores a la tarea 4, se identificaron cinco tipos de configuraciones que hemos denominado: 1) analítica - extensiva; 2) analítica intensiva; 3) trazado de tangentes; 4) uso de situaciones particulares de variación; y 5) límite de las tasas medias de variación. Para cada una de estas configuraciones cognitivas, analizamos un ejemplo prototípico.

\subsubsection{Configuración Cognitiva 1: analítica-extensiva}

El eje central de este tipo de resolución son las argumentaciones o justificaciones a la proposición aludida por el elemento lingüístico la derivada de una función constante siempre es igual a cero, mediante casos particulares del cálculo de las pendientes de algunas rectas horizontales. La Figura 3 muestra la solución dada por un estudiante (estudiante A) a la tarea 4.

\section{Prácticas}

Como puede observarse en la Figura 3, la actividad matemática desarrollada por el estudiante A comienza con la representación (simbólica y gráfica) de la función constante $\mathrm{f}(\mathrm{x})=2$. Luego, considerando la derivada como la pendiente de la recta tangente a la función que plantea, calcula la pendiente de la recta tangente a la función $\mathrm{f}(\mathrm{x})=2$ dados los puntos $(3,2),(4,2)$, aplicando que $\mathrm{m}=\frac{2-2}{4-3}$. El estudiante A finaliza su actividad considerando que 
lo anterior justifica la proposición la derivada de una función constante siempre es igual a cero.

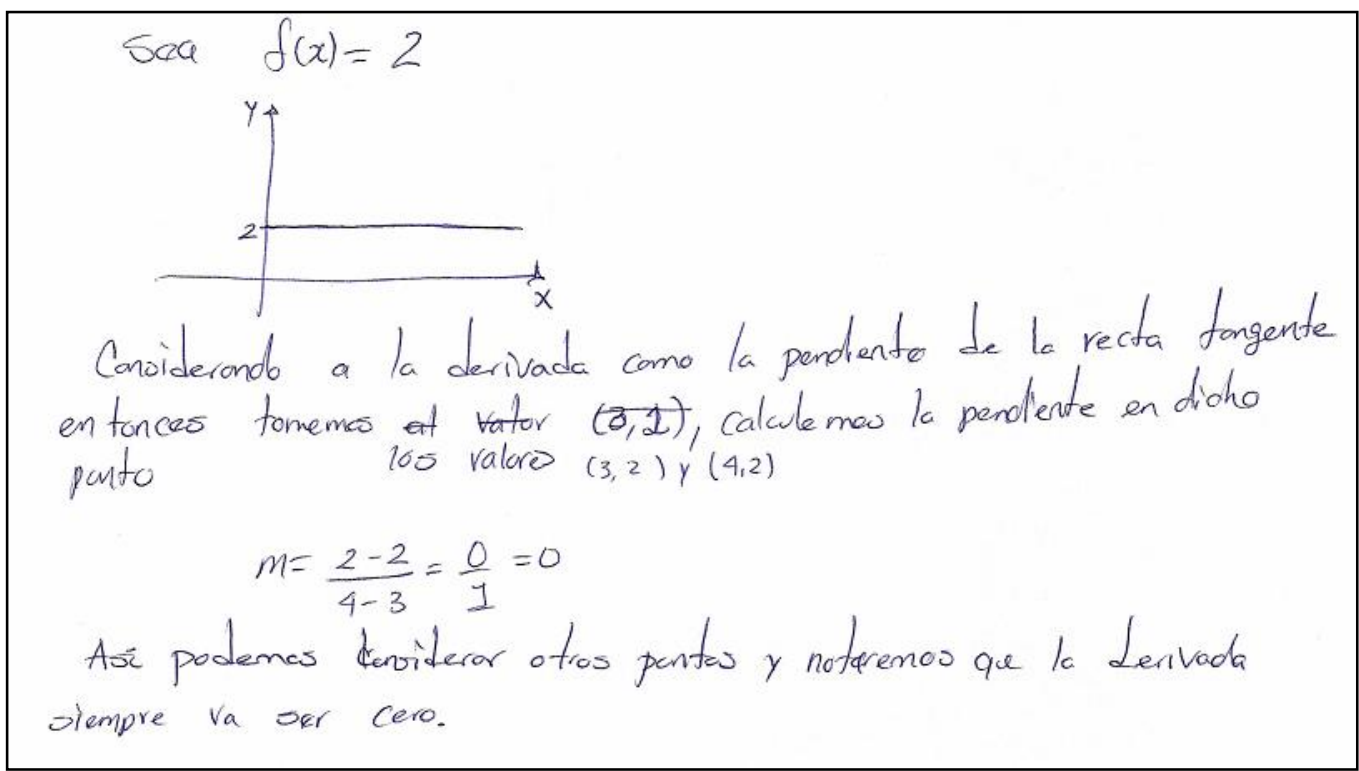

Figura 3 - Solución a la tarea 4 por el estudiante A

\section{Configuración Cognitiva}

En la actividad matemática desarrollada por el estudiante A, es posible identificar diversos elementos lingüísticos (gráficos, simbólicos y verbales) que dan cuenta de conceptos/definiciones y proposiciones que el estudiante utiliza en sus procedimientos y en sus argumentos. Ejemplo de elementos lingüísticos son: la representación gráfica y simbólica para la función constante $f(x)=2$, el elemento lingüístico verbal Considero a la derivada como la pendiente de la recta tangente, la representación simbólica para el cálculo de la pendiente, entre otros.

Entre los conceptos/definiciones centrales se encuentra el de función constante, particularizada en $f(x)=2$, y derivada, entendida como pendiente de la recta tangente, referida por el estudiante mediante la expresión considero a la derivada como pendiente de la recta tangente.

En cuanto a las proposiciones/propiedades destacan la relación analítica $y-y_{1}=$ $m\left(x-x_{1}\right)$, particularizada en $m=\frac{2-2}{4-3}$ y las proposiciones implícitas la pendiente de una secante a una línea recta coincide con la pendiente de la recta, $m=0$, y la derivada de la función $f(x)=2$ es cero.

Los conceptos y proposiciones centrales, referidos anteriormente, son utilizados en un procedimiento analítico en el cual se calcula la pendiente de la recta tangente, dados dos puntos cuyas coordenadas son $((3,2)$ y $(4,2))$ de la recta $f(x)=2$. Este procedimiento lo 
hace reiterativo cuando señala así podemos considerar otros puntos..., y considera que lo anterior justifica la proposición la derivada de una función constante siempre es igual a cero, cuando continúa ...y notaremos que la derivada siempre va a ser cero. Sin embargo, el estudiante A no logra justificar la proposición general inicial y resuelve la tarea para el caso particular $f(x)=2$.

En la solución que presenta el estudiante A, se observa un proceso de particularización del problema, ya que traslada la tarea inicial de justificar la proposición la derivada de una función constante siempre es igual a cero a justificar la proposición la derivada de la función $f(x)=2$ siempre es igual a cero.

Adicionalmente se encuentra que el estudiante A no resolvió el apartado b) de la Tarea 4, lo cual evidencia escaso conocimiento común y, al parecer, la ausencia de conocimiento especializado del contenido para su solución.

Debido a limitaciones de espacio, el análisis de las restantes configuraciones cognitivas identificadas lo realizamos de forma menos detallada; es decir, se describen las prácticas matemáticas, señalando los objetos primarios y los procesos matemáticos más relevantes.

\subsubsection{Configuración cognitiva 2: analítica-intensiva}

La Figura 4 muestra la solución dada por el estudiante B a la Tarea 4, la cual hemos inscrito dentro del tipo analítica-intensiva. A diferencia del tipo de la solución anterior, las soluciones analíticas-intensivas tienen la característica de que las argumentaciones o justificaciones de la proposición a la que hace referencia la expresión la derivada de una función constante siempre es igual a cero, se realiza mediante el cálculo de la pendiente de una función constante genérica .

En la Figura 4 observamos que el estudiante, primeramente, proporciona la gráfica de una función constante cualquiera y considera dos puntos cualesquiera de ella $\left(x_{1}, f\left(x_{1}\right)\right)$ y $\left(x_{2}, f\left(x_{2}\right)\right)$. Seguidamente calcula la pendiente, dados dos puntos, de la recta horizontal que representa la función constante, pues considera que la derivada de la función constante es la pendiente de la recta tangente. El estudiante concluye proporcionando la representación gráfica de la función derivada.

En la solución que presenta el estudiante B, pueden identificarse conceptos centrales tales como la función constante genérica, referida por el elemento lingüístico gráfico y 
simbólico $f(x)=k$, y la función derivada referida por los elementos lingüísticos $f^{\prime}(x)=$ pendiente de la recta tangente a la curva en el punto $x=x_{0}$ y la gráfica de la recta que coincide con el eje $x$. Los puntos $x_{1}$ y $x_{2}$, señalados en la primera gráfica, junto con la propiedad $m=\frac{y_{2}-y_{1}}{x_{2}-x_{1}}$ utilizada como $f^{\prime}(x)=\frac{k-k}{x_{1}-x_{2}}$, muestran evidencia del procedimiento analítico seguido, el cual consistió en el cálculo de la pendiente de una recta horizontal cualquiera dados dos puntos cualesquiera pertenecientes a la misma. No queda explícito que el alumno sea consciente de la proposición implícita la pendiente de la secante a una recta coincide con la pendiente de la tangente a dicha recta, y ambas coinciden con la pendiente de dicha recta, lo cual justificaría la solución dada.

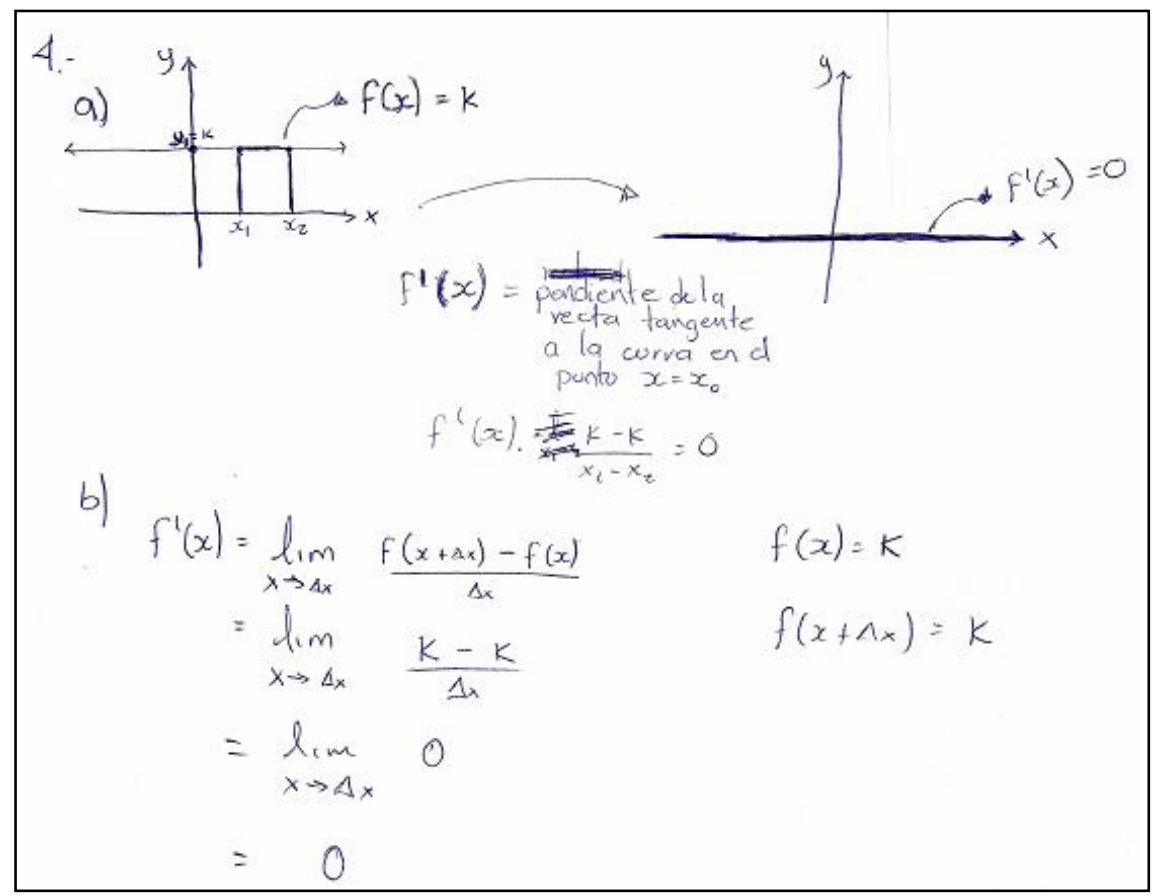

Figura 4 - Solución a la tarea 4 por el estudiante B

Además, se observa que el estudiante no justifica el uso de los elementos lingüísticos, conceptos, proposiciones y procedimientos utilizados en su solución, o bien, considera que el conjunto de todos estos son la justificación. De cualquier forma, el estudiante B, de hecho, opera con una función cualesquiera, pero omite explicitar que su razonamiento es válido para cualquier función constante.

En cuanto al apartado b) de la tarea, si bien es cierto que el estudiante escribe limite cuando $x$ tiende $\Delta x$, tal error parece no afectar a sus consideraciones en relación con el valor del límite y con sus subsecuentes consideraciones. Vemos que dicho estudiante no tuvo dificultad en probar la proposición inicial mediante la derivada como límite del cociente de incrementos. La actividad matemática desarrollada por el estudiante B en la Tarea 4 sugiere 
que dicho estudiante posee un grado de conocimiento común suficiente, en tanto que fue capaz de resolver la tarea; así mismo, evidencia cierto grado de conocimiento especializado del contenido, ya que planteó dos procedimientos y justificaciones distintas para la proposición planteada.

\subsubsection{Configuración cognitiva 3: trazado de rectas tangentes}

La Figura 5 muestra un ejemplo característico de este tipo de solución. La característica central son las justificaciones dadas a la proposición referida por la expresión la derivada de una función constante siempre es igual a cero, a partir del trazado de rectas tangentes a la función constante, lo cual se evidencia con la solución dada por el estudiante C.

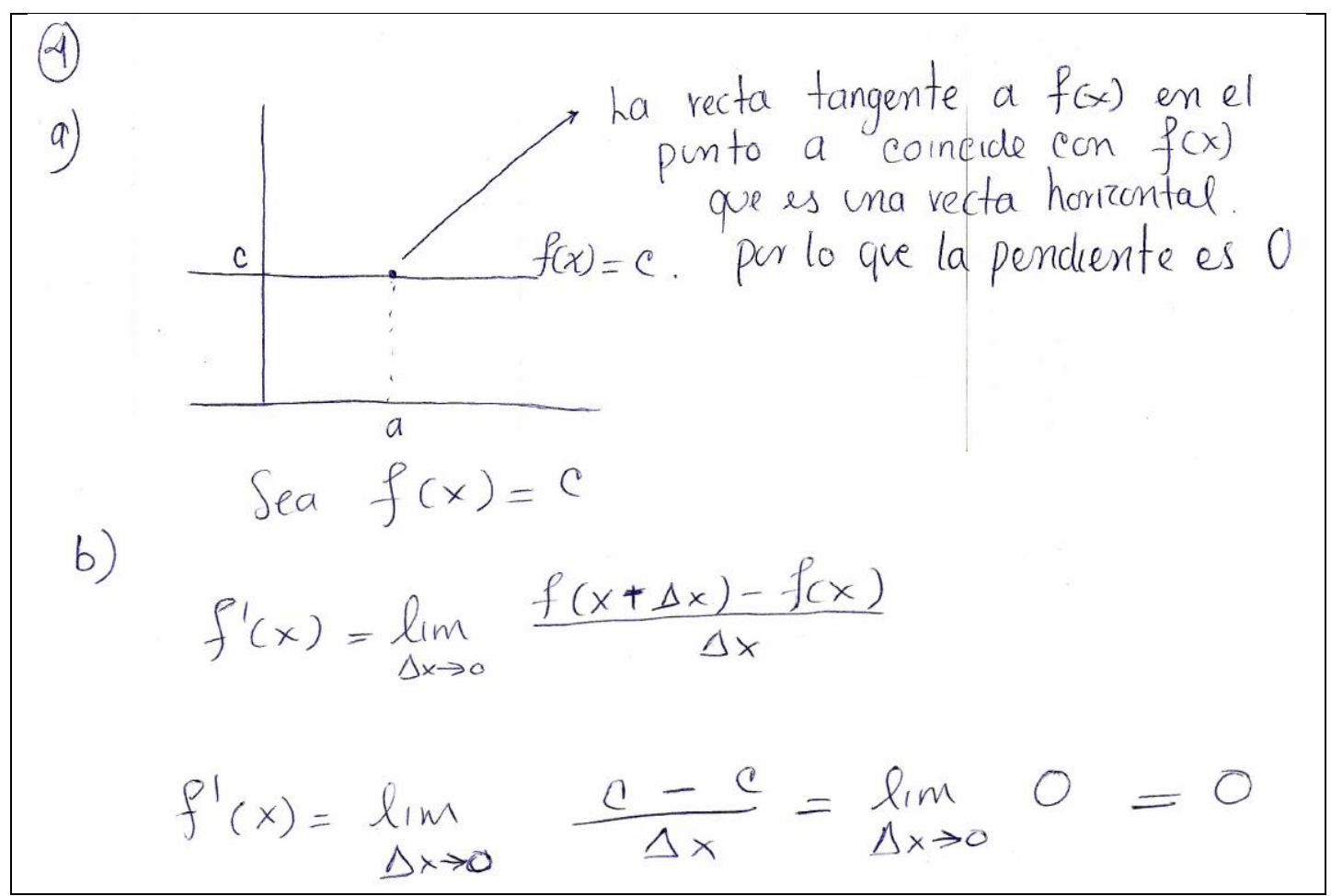

Figura 5 - Solución de la tarea 4 por el estudiante C

Como se puede observar en la Figura 5, la solución del estudiante C, a diferencia de los dos tipos anteriores, podría considerarse como más visual. Se identifican elementos lingüísticos gráficos y verbales (apartado a) que hacen referencia a conceptos centrales tales como función constante $\mathrm{f}(\mathrm{x})=\mathrm{c}$ y derivada, entendida como pendiente de la recta tangente.

Así mismo, el estudiante utiliza elementos lingüísticos que refieren a proposiciones tales como: [la gráfica de] la recta tangente a la función $f(x)$ en el punto a coincide con la [gráfica de] la función $f(x)$, y la pendiente de una recta horizontal es cero. Estas 
proposiciones dan cuenta del procedimiento y su justificación empleados por el estudiante para la resolución de la tarea. Así, el procedimiento consiste en el trazo de rectas tangentes a la función constante en puntos cualesquiera pertenecientes a dicha función, y la justificación es: dado que la representación gráfica de las rectas tangentes coinciden con la representación gráfica de la función, y tomando en cuenta que la pendiente de una recta horizontal es cero, entonces la pendiente de la recta tangente a la función constante es cero.

El apartado b) de la tarea es resuelto mediante la manipulación algebraica de elementos simbólicos que hacen referencia a la definición de la derivada, presentada frecuentemente en los libros de texto.

Sobre la base de lo anterior, concluimos que el estudiante $\mathrm{C}$ posee el conocimiento común suficiente, que es necesario para resolver la tarea. Sin embargo proponer una solución visual (apartado a) y no realizar conexiones con otros objetos matemáticos para justificar de manera más formal la proposición inicial, evidencia que su conocimiento especializado del contenido requerido para dar solución a la tarea debe ser mejorado.

\subsubsection{Configuración cognitiva 4: situaciones particulares de variación}

A diferencia de los tres tipos de configuraciones anteriores, en ésta existe un cambio sustancial: la consideración de la derivada como velocidad. De esta forma, la característica central es la justificación de la proposición mediante situaciones concretas de variación, específicamente, de velocidad. La Figura 6 muestra la solución presentada por un estudiante y que es prototípica de las soluciones mediante situaciones particulares de variación.

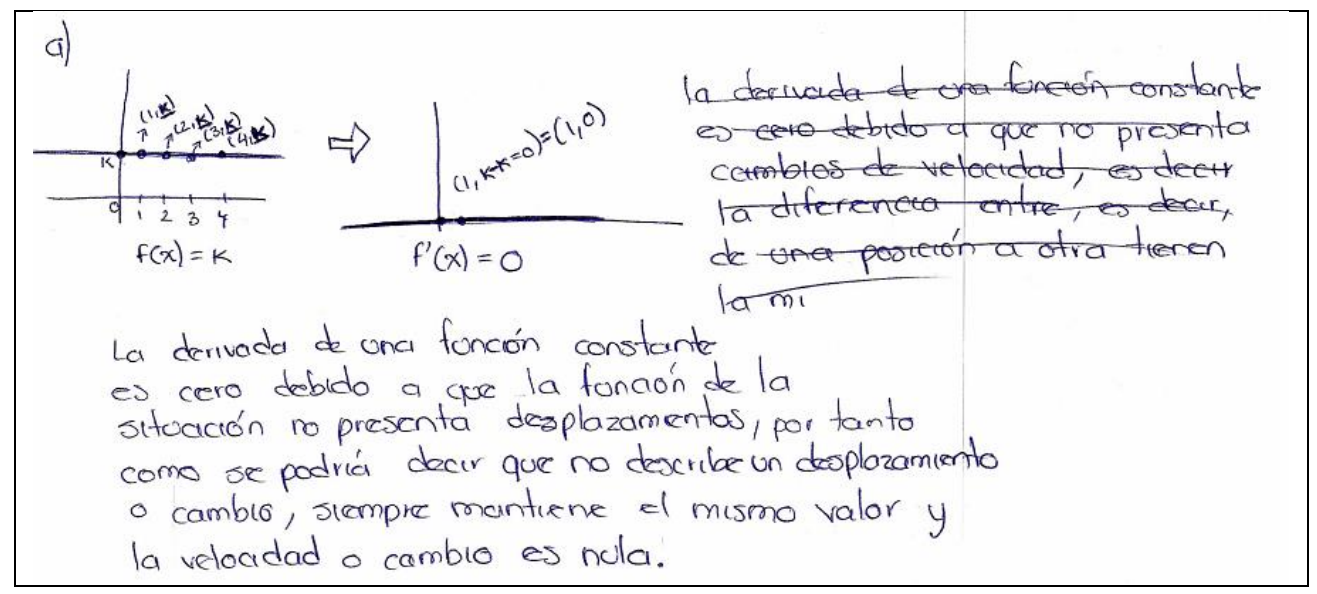




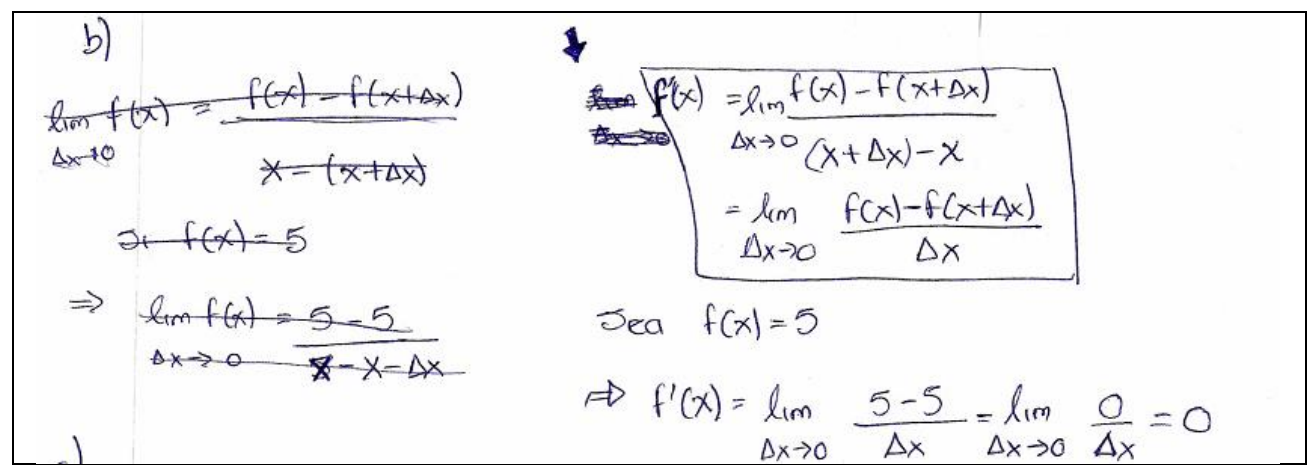

Figura 6 - Solución de la tarea 4 por el estudiante D

A partir de los elementos lingüísticos (gráficos, verbales y simbólicos) de la solución del estudiante $\mathrm{D}$, podemos observar que considera conceptos tales como función constante arbitraria $f(x)=k$, distancia o desplazamiento, tiempo y derivada la cual es interpretada como la función velocidad. Aunque se percibe una aparente desconexión entre el concepto de función constante y el de desplazamiento cuando señala la derivada de una función constante es cero debido a que la función de la situación no presenta desplazamientos..., utiliza la función constante como función de desplazamiento de algún objeto al que nunca hace referencia. Sin embargo, el estudiante D parece no ser consciente de esta relación.

Se puede decir que el procedimiento seguido por el estudiante D es empíricodescriptivo y, en él, se encuentra implícita una proposición fundamental para la solución de la tarea si un objeto no se desplaza a lo largo del tiempo, entonces la velocidad del objeto es nula. Sobre la base de dicha proposición y de los objetos matemáticos antes mencionados, el estudiante argumenta “...por tanto como se podría decir que no describe un desplazamiento o cambio [la función constante], siempre mantiene el mismo valor y la velocidad o cambio es nula" (ESTUDIANTE D), lo cual para él justifica la proposición inicial y por tanto da solución al apartado a) de la tarea.

En el apartado b) observamos que el estudiante D evidencia dificultades para utilizar la definición formal de la derivada como límite del cociente de incrementos, propone la expresión $\lim _{\Delta x \rightarrow 0} f(x)=\lim _{\Delta x \rightarrow 0} \frac{f(x)-f(x+\Delta x)}{x-(x+\Delta x)}$, que corresponde a una definición equivalente de la derivada tomando el incremento $\Delta x$ en un intervalo donde la función es decreciente; sin embargo, no parece claro que el estudiante sea consciente de esta propiedad. Posteriormente, propone la siguiente expresión $f^{\prime}(x)=\lim _{\Delta x \rightarrow 0} \frac{f(x)-f(x+\Delta x)}{(x+\Delta x)-x}$. Finalmente, realiza un proceso de particularización de la tarea propuesta, tomando una función constante particular, y prueba que la derivada de la función $f(x)=5$ es cero. 
Es plausible inferir que el estudiante D tiene dificultades para manifestar tanto el conocimiento especializado del contenido como el conocimiento común requerido para la solución de la tarea.

\subsubsection{Configuración cognitiva 5: límite de tasas medias de variación}

La Figura 7 muestra la solución dada por el estudiante $\mathrm{E}$ a la tarea 4 y que es característica de este quinto tipo de configuración cognitiva.

Una de las características que diferencia este tipo de solución respecto del anterior es el uso de argumentaciones basadas en la noción de tasa instantánea de variación, sin considerar casos concretos como la velocidad. Así, en la Figura 7 puede observarse cómo el estudiante $\mathrm{E}$ usa un elemento lingüístico gráfico el cual hace referencia a los conceptos de función constante arbitraria $\mathrm{f}(\mathrm{x})=\mathrm{k}$, valores del dominio (a y b del eje $\mathrm{x}$ ), imagen ( $\mathrm{f}(\mathrm{a}) \mathrm{y}$ $\mathrm{f}(\mathrm{b})$ ) y la noción de cambio (la cual interpreta por medio de la relación $\frac{\mathrm{f}(\mathrm{b})-\mathrm{f}(\mathrm{a})}{\mathrm{b}-\mathrm{a}}$ ). La derivada la interpreta como razón instantánea de cambio cuando señala como la derivada se calcula con los cambios en la función, entonces si nos situamos en dos puntos $x=a$ y $x=b y$ calculamos la razón $\lim _{b \rightarrow a} \frac{f(b)-f(a)}{b-a}$ se obtiene... (Solución apartado b).

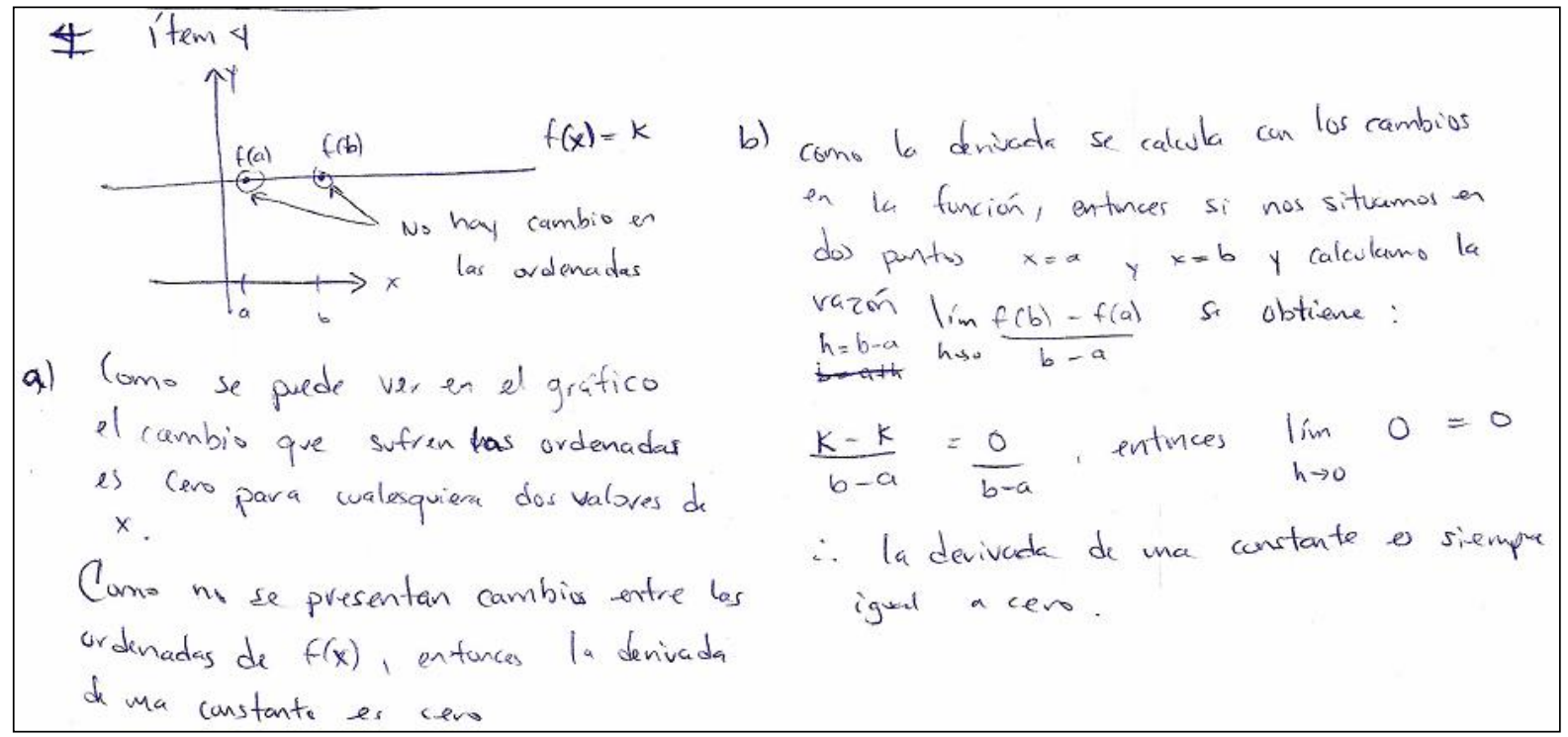

Figura 7 - Solución de la tarea 4 por el estudiante E

Luego el estudiante da evidencia del procedimiento usado al describir la siguiente proposición como se puede ver en el gráfico el cambio que sufren las ordenadas $\left[\frac{\mathrm{f}(\mathrm{b})-\mathrm{f}(\mathrm{a})}{\mathrm{b}-\mathrm{a}}\right]$ es cero para cualquiera dos valores de $x$. El estudiante observa el cambio que sufren dos 
ordenadas (imágenes) respecto al cambio de sus abscisas, para posteriormente tomar el paso al límite (solución dos) que denota la razón instantánea de cambio. El estudiante E argumenta: "como no se presentan cambios entre las ordenadas de $f(x)$, entonces la derivada de una constante es cero". Lo anterior se puede expresar mediante la proposición implícita dado que el cambio es nulo entonces la razón instantánea de cambio también es nula $\left(\mathrm{si} \frac{\mathrm{f}(\mathrm{b})-\mathrm{f}(\mathrm{a})}{\mathrm{b}-\mathrm{a}}=0\right.$ entonces $\left.\lim _{\mathrm{b} \rightarrow \mathrm{a}} \frac{\mathrm{f}(\mathrm{b})-\mathrm{f}(\mathrm{a})}{\mathrm{b}-\mathrm{a}}=0\right)$.

La solución dada por el profesor en formación muestra evidencia del dominio de objetos matemáticos primarios centrales (elementos lingüísticos, conceptos, proposiciones, procedimientos y justificaciones) alrededor de la noción de la derivada como razón instantánea de cambio. De esta forma, es claro que el futuro profesor posee un buen dominio del conocimiento común, y muestra evidencia de cierto grado de conocimiento especializado del contenido, ambos requeridos para la solución de la tarea.

\subsection{Configuraciones cognitivas asociadas a la resolución de la tarea 8}

Mediante las soluciones que los futuros profesores de bachillerato dieron a la tarea 8 , se pudieron identificar 4 tipos distintos de configuraciones que hemos denominado: 1) patrón numérico; 2) uso de la relación física $v=\mathrm{d} / \mathrm{t}$; 3) aproximación por la izquierda o derecha, y 4) aproximación bilateral. Es importante señalar que, inicialmente, esperábamos (a excepción de la opción a, Figura 2), respuestas parecidas a las reportadas en el trabajo de Çetin (2009), en donde los estudiantes resolvieron la tarea mediante los siguientes procedimientos:

- Sumando las distancias entre un tiempo y otro y dividiendo el resultado por seis, es decir, $\frac{1}{6}[(\mathrm{f}(0.1)-\mathrm{f}(0))+(\mathrm{f}(0.2)-\mathrm{f}(0.1))+\cdots+(\mathrm{f}(0.6)-\mathrm{f}(0.5))]$, lo que da como resultado la opción b.

- Sumando las imágenes de cada uno de los tiempos dados y dividiendo el resultado por siete, es decir, $\frac{1}{7}[\mathrm{f}(0)+\mathrm{f}(0.1)+\cdots+\mathrm{f}(0.6)]$, lo que da como resultado la opción c.

- Eligiendo la imagen de $t=0.4, \mathrm{f}(0.4)$, como la velocidad puntual de la pelota, lo que resulta en la opción d.

Sin embargo, los cuatro tipos de configuraciones que identificamos tienen características distintas. A continuación, procediendo como en la sección 3.1, analizamos cada 
uno de dichos tipos de configuraciones mediante un ejemplo característico para cada una de las tipologías.

\subsubsection{Configuración cognitiva 1: patrón numérico}

La característica de este tipo de solución, aunque los estudiantes que la propusieron no llegaron a concretarla, es que a partir de los datos numéricos presentados en la tabla (puntos pertenecientes a una función), se intenta determinar el patrón o regla de correspondencia que define la función. La Figura 8 presenta una de las respuestas (estudiante F) que ilustra este tipo de solución.

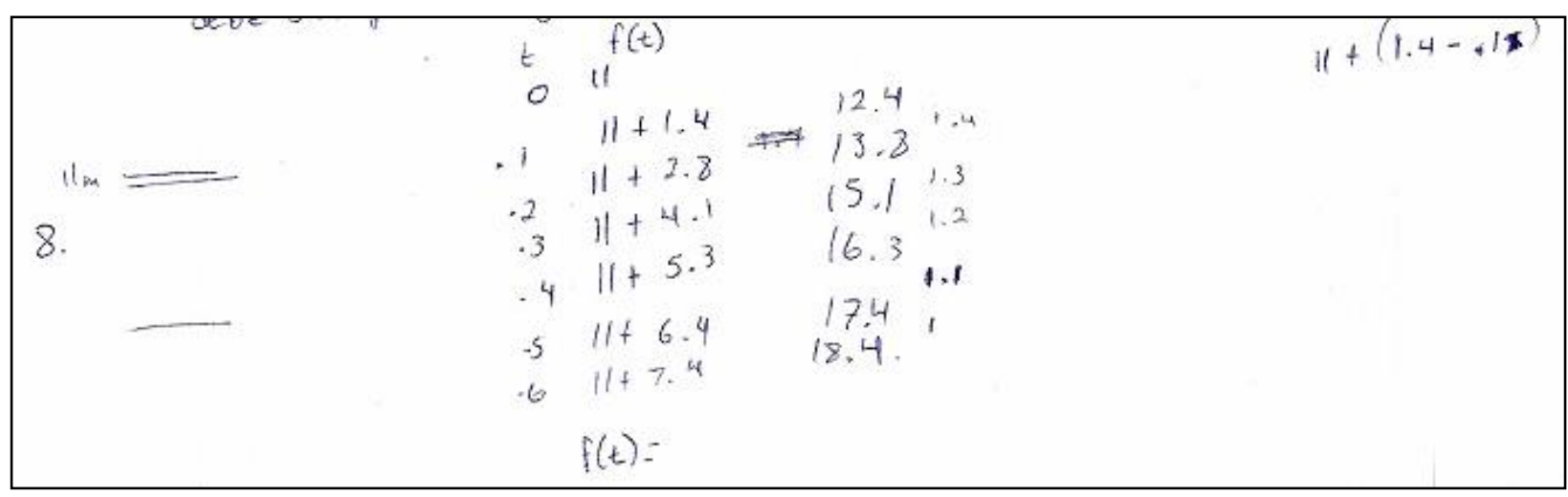

Figura 8 - Solución de la tarea 8 por el estudiante F

Puede observarse que el estudiante $\mathrm{F}$ utiliza elementos lingüísticos que hacen referencia a números o cantidades que representan tanto al tiempo transcurrido $\mathrm{t}=$ $0,0.1, \ldots, 0.6$ como a la altura de la pelota para un tiempo t determinado $f(t)=11,11+$ $1.4, \ldots, 11+7.4$. Luego, haciendo uso de la proposición una pelota se lanza al aire desde un puente de 11 metros de altura $[f(t)=11$ para $t=0]$, realiza una descomposición de las alturas $\mathrm{f}(\mathrm{t}) \quad 12.4=11+1.4,13.8=11+2.8, \ldots, 18.4=11+7.4$. Luego, procede por ensayo y error para encontrar un patrón que le ayude a determinar la expresión para f(t), lo cual se evidencia con la expresión $11+(1.4-.1)$. El estudiante $\mathrm{F}$ no logra concretar su solución.

Al parecer, este estudiante no se percata de que el procedimiento que ha usado para resolver la tarea es, quizá, el más complicado de todos los posibles. Tratar de encontrar una expresión simbólica para $\mathrm{f}(\mathrm{t})$ - dado que los datos de la tabla no se ajustan de manera directa al modelo físico-matemático descrito en el apartado 2.2 - a partir de los siete puntos dados, es una tarea complicada. Es posible encontrar una función que se comporte aproximadamente 
igual a lo largo de esos siete puntos; no obstante, esta tarea es difícil puesto que se refiere a una función polinómica de, al menos, grado seis, y los participantes del estudio no contaban con calculadoras graficadoras, ni algún otro tipo de tecnología que facilitara este trabajo.

Una posible solución por esta vía - en lápiz y papel sin el uso de algún recurso podría realizarse haciéndose uso de la interpolación polinómica de Lagrange, la cual señala que dado un conjunto de $\mathrm{k}+1$ puntos $\left(\mathrm{x}_{0}, \mathrm{y}_{0}\right), \ldots,\left(\mathrm{x}_{\mathrm{k}}, \mathrm{y}_{\mathrm{k}}\right)$, donde todos los $\mathrm{x}_{\mathrm{j}}$ se asumen distintos, es posible hallar una función, que pase por ellos, mediante la combinación lineal:

$$
L(x)=\sum_{j=0}^{k} y_{j} \ell_{j}(x)
$$

donde $y_{j}$ son escalares y $\ell_{j}(x)$ viene dada por:

$$
\ell_{j}(x)=\prod_{i=0}^{k} \frac{x-x_{i}}{x_{j}-x_{i}}
$$

Así, el polinomio interpolador para nuestra tarea sería:

$$
L(t)=\frac{25000 t^{6}-52500 t^{5}+43750 t^{4}-18375 t^{3}+3160 t^{2}+2349 t+1980}{180}
$$

La función de velocidad de la pelota para un tiempo $t \in[0,0.6]$

$$
L^{\prime}(t)=\frac{150000 t^{5}-262500 t^{4}+175000 t^{3}-55125 t^{2}+6320 t+2349}{180}
$$

Y finalmente, la velocidad de la pelota en $t=0.4$ es

$$
L^{\prime}(0.4)=\frac{691}{60} \approx 11.5166 \mathrm{~m} / \mathrm{s}
$$

\subsubsection{Configuración cognitiva 2: uso de la relación física $v=d / t$}

La Figura 9 muestra la solución que da el estudiante $G$ a la tarea 8 y que es característica de este tipo de configuración.

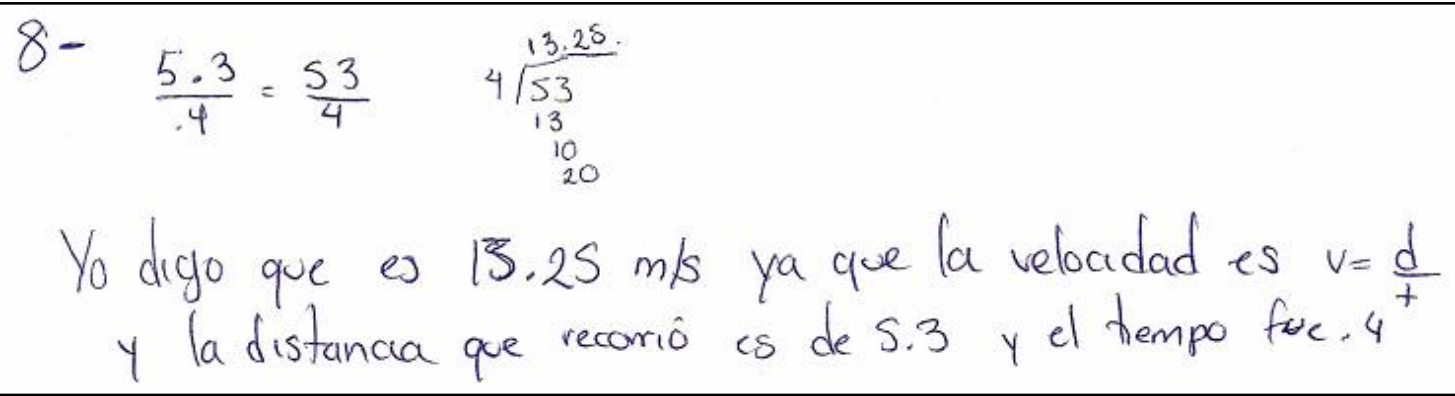

Figura 9 - Solución de la tarea 8 por el estudiante G 
Como se puede observar en la Figura 9, la característica central de este tipo de solución es el empleo tanto de la proposición velocidad es igual a distancia entre tiempo $(\mathrm{v}=\mathrm{d} / \mathrm{t})$ como de la desconsideración que se realiza del objeto derivada como velocidad instantánea.

Así, el estudiante evidencia elementos lingüísticos simbólicos y verbales que evocan a la consideración de conceptos tales como velocidad (promedio), distancia, tiempo; y proposiciones tales como ' $\mathrm{v}=\mathrm{d} / \mathrm{t}$ ' $\mathrm{y}$ 'Yo digo que es $13.25 \mathrm{~m} / \mathrm{s}$....'

El procedimiento usado fue el cálculo de la velocidad promedio de la pelota entre $\mathrm{t}=0 \mathrm{y} \mathrm{t}=0.4$, mediante la relación $\mathrm{v}=\mathrm{d} / \mathrm{t}$. Así, el estudiante $\mathrm{G}$ halla el tiempo transcurrido entre $\mathrm{t}=0 \mathrm{y} \mathrm{t}=0.4$ 'el tiempo fue de $0.4[0.4 \mathrm{~s}-0 \mathrm{~s}]$ ' y la distancia que recorre la pelota en ese lapso 'la distancia que recorrió es de 5.3 [16.3m - 11m]'. Luego, calcula la velocidad promedio $\frac{5.3}{0.4}=\frac{53}{4}$ y da su respuesta mediante la expresión $13.25 \mathrm{~m} / \mathrm{s}$ ", la cual justifica "ya que la velocidad es $\mathrm{v}=\mathrm{d} / \mathrm{t}$ y la distancia que recorrió [la pelota] es de $5.3 \mathrm{y}$ el tiempo fue de $0.4 "$ (ESTUDIANTE G).

El estudiante $\mathrm{G}$ parece no percatarse de que la tarea requiere la interpretación de la derivada en un punto como velocidad instantánea. Tampoco tiene en cuenta la relación entre velocidad promedio $(\mathrm{v}=\mathrm{d} / \mathrm{t})$ y la pendiente de una recta secante que corta a la función en $\mathrm{t}=0 \mathrm{y} \mathrm{t}=0.4$, relación que le habría ayudado a reconocer que su respuesta era incorrecta. Con lo anterior, parece argumentado que el estudiante $G$ no muestra evidencia del conocimiento matemático ampliado (PINO-FAN; GODINO; FONT, 2013) requerido para resolver la tarea.

\subsubsection{Configuración cognitiva 3: aproximación por la izquierda o derecha}

Este tipo de configuración tiene muchas similitudes con el tipo anterior. Entre las distinciones más notables se encuentra el hecho de que los estudiantes no consideran (al menos explícitamente) la relación $v=d / t$, y la otra es que para calcular la velocidad promedio se tomaron valores más próximos a $t=0.4 \mathrm{~s}$ (por ejemplo $\mathrm{t}=0.3 \mathrm{~s}$ ). La Figura 10 muestra la solución que da el estudiante $\mathrm{H}$ y que es prototípica de este tipo de configuración. 


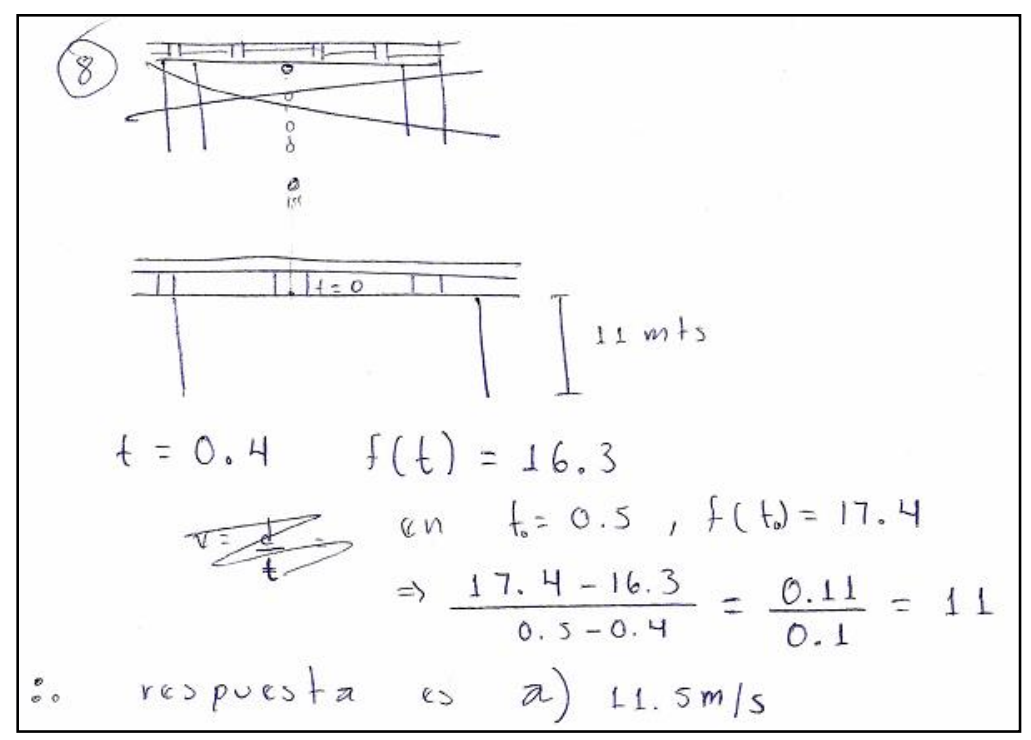

Figura 10 - Solución de la tarea 8 del estudiante $\mathrm{H}$

En la solución que se presenta en la Figura 10, se observan elementos lingüísticos icónicos (dibujo del puente) y simbólicos que dan evidencia tanto de conceptos tales como distancia, tiempo y velocidad promedio; como de proposiciones tales como la pelota se lanza al aire desde un puente de 11 metros de altura $[\mathrm{f}(\mathrm{t})=11$ para $\mathrm{t}=0]$ y velocidad es igual a distancia entre tiempo $(v=d / t)$. A partir de estos objetos matemáticos, el estudiante $\mathrm{H}$ desarrolla un procedimiento centrado en el cálculo de la velocidad promedio de la pelota entre los tiempos $\mathrm{t}=0.4 \mathrm{y} \mathrm{t}=0.5 \mathrm{y}$ considerando un incremento $\mathrm{h}=0.1$ (más pequeño que en el tipo de solución anterior) $\frac{17.4-16.3}{0.5-0.4}=\frac{0.11}{0.1}=11$.

A pesar del error en el que incurre el estudiante, al establecer que el resultado de la resta 17.4-16.3=0.11, afirmamos que su solución es una aproximación por la derecha a la velocidad de la pelota en el instante $t=0.4$, ya que considera un tiempo posterior $(t=0.5)$ para el cálculo de la velocidad promedio; y dicho error no afecta a la globalidad de su razonamiento ni de los objetos matemáticos primarios que moviliza en su solución. En este caso, el procedimiento sí que es usado por el estudiante como argumentación para su respuesta. La afirmación, por tanto la respuesta es a) $11.5 \mathrm{~m} / \mathrm{s}$, la propone debido a que es la opción más parecida al resultado que ha obtenido con sus cálculos.

Al igual que en el caso anterior, no se tiene evidencia suficiente de que el estudiante $H$ se haya percatado de que en realidad ha calculado la velocidad promedio de la pelota (entre $t=0.4$ y $t=0.5$ ) y no su velocidad instantánea en el instante $t=0.4$. 


\subsubsection{Configuración cognitiva 4: aproximación bilateral}

La característica más importante en esta configuración es la aproximación bilateral que se realiza a la derivada de la función en el punto $t=0.4$, a través de valores numéricos de la función. Lo anterior también se conoce como derivada numérica. La Figura 11 muestra la solución dada por el estudiante I, que ilustra este tipo de configuración cognitiva.

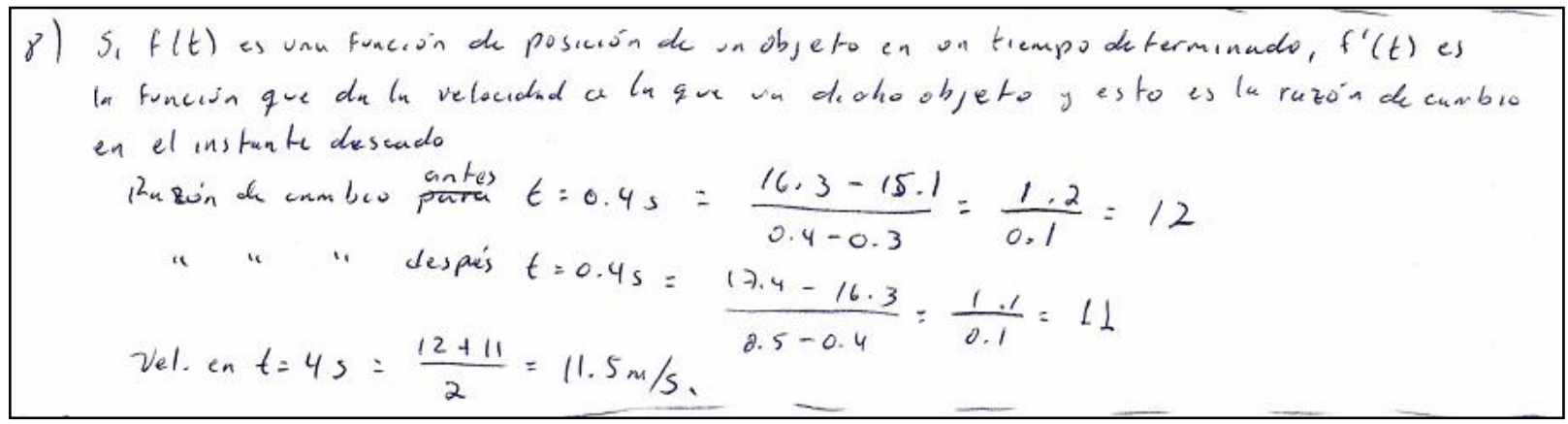

Figura 11 - Solución a la tarea 8 por el estudiante I

El estudiante I comienza la solución de la tarea indicando "Si $f(t)$ es una función de posición de un objeto en un tiempo determinado, $f^{\prime}(t)$ es la función que da la velocidad a la que va dicho objeto y esto es la razón de cambio en el instante deseado. De esta primera expresión dada por el estudiante, se puede inferir el dominio de conceptos centrales tales como función (posición de un objeto respecto del tiempo), derivada (como función velocidad), y la derivada en un punto interpretada como razón de cambio instantánea. También aparecen proposiciones tales como $f(t)$ es una función de posición de un objeto en un tiempo determinado y $f^{\prime}(t)$ es la función que da la velocidad a la que va dicho objeto y una última que da cuenta de la comprensión que tiene el estudiante de la tarea "...y esto es la razón de cambio en el instante deseado". Esta última proposición sugiere que pretende hallar la razón de cambio en el instante $t=0.4$. Esto último se hace más evidente a partir del elemento lingüístico que revela el procedimiento que sigue.

Primero considera una razón de cambio antes de $t=0.4 \mathrm{~s}$ que se refiere al cálculo de la razón de cambio o velocidad promedio de la pelota entre los tiempos $t=0.3$ y $t=0.4$ $\frac{16.3-15.1}{0.4-0.3}=\frac{1.2}{0.1}=12$ (aproximación a la razón de cambio en el instante $t=0.4$ por valores de la izquierda y con incremento $\mathrm{h}=0.1$ ). Luego considera una aproximación 'después de $t=0.4 s^{\prime}$, que hace referencia al cálculo de la razón de cambio o velocidad promedio de la pelota entre los tiempos $\mathrm{t}=0.4 \mathrm{y} \mathrm{t}=0.5 \frac{17.4-16.3}{0.5-0.4}=\frac{1.1}{0.1}=11$ (aproximación a la razón de 
cambio en el instante $t=0.4$ por valores de la derecha y con incremento $h=0.1$ ). A partir de estas velocidades promedio el estudiante I calcula la razón de cambio instantánea para $t=0.4$

velocidad en $\mathrm{t}=0.4 \mathrm{~s}=\frac{12+11}{2}=11.5 \mathrm{~m} / \mathrm{s}$ (aproximación bilateral a la velocidad de la pelota en el instante $t=0.4 \mathrm{~s}$, mediante el promedio de las velocidades promedio, con $\mathrm{t}=0.4$ en el centro del intervalo considerado).

Es preciso indicar que tanto las tareas como los análisis que aquí se realizan corresponden al contexto y a los objetivos de esta investigación. En relación con el análisis epistémico realizado, previo a la implementación del cuestionario, éste se podría ampliar y evidenciar en otros trabajos dando cuenta de aspectos relacionados con el dominio de la función, con la validez de la afirmación la derivada de una función constante siempre es igual a cero, referida a funciones de valor y variable real, y a la topología usual del campo numérico de los números reales. Lo mismo se puede afirmar de la segunda tarea, en tanto que se podrían considerar aspectos tales como: la derivabilidad de la función representada numéricamente por la tabla, la posibilidad que la componente horizontal de la velocidad no sea cero, resistencia del aire etc. Ahora bien, no parece necesaria esta ampliación dada la población a la cual se ha aplicado el cuestionario y los objetivos que perseguimos en este artículo.

\section{Resultados y Discusión}

Para el análisis de las tareas respecto a la variable grado de corrección, consideramos los siguientes casos: respuesta correcta, respuesta parcialmente correcta, respuesta incorrecta y no responden. Para el caso de la tarea 4 (Figura 1), se consideraron como correctas aquellas respuestas en las que se emplearon representaciones gráficas y descripciones verbales para dar justificaciones válidas para la proposición la derivada de una función constante siempre es cero. Respuestas parcialmente correctas eran aquellas en las que se proporcionaban gráficas válidas, pero las descripciones verbales no justificaban la proposición inicial. Se consideraron respuestas incorrectas aquellas en las que no se proporcionaron ni gráficas, ni descripciones verbales válidas, para justificar la proposición en cuestión. La Tabla 1 presenta los resultados obtenidos para el grado de corrección de la tarea 4. 
Tabla 1 - Frecuencias y porcentajes para el grado de corrección de la Tarea 4

\begin{tabular}{|c|c|c|c|c|}
\hline \multirow{2}{*}{ Grado de Corrección } & \multicolumn{2}{|c|}{ Apartado a) } & \multicolumn{2}{|c|}{ Apartado b) } \\
\hline & Frecuencia & Porcentaje & Frecuencia & Porcentaje \\
\hline Correcta & 19 & 35,9 & 23 & 43,4 \\
\hline Parcialmente correcta & 21 & 39,6 & 8 & 15,1 \\
\hline Incorrecta & 8 & 15,1 & 15 & 28,3 \\
\hline No responden & 5 & 9,4 & 7 & 13,2 \\
\hline Total & 53 & 100 & 53 & 100 \\
\hline
\end{tabular}

Como puede observarse en la Tabla 1, sólo el 35,9\% de los futuros profesores resolvió de forma correcta el apartado a) de la tarea 4. Esto sugiere que más de la mitad de los estudiantes para profesor presentaron dificultades para resolver dicho ítem. El apartado b) lo responden correctamente el $43,4 \%$ de los estudiantes, lo que evidencia que más de la mitad de los futuros profesores tienen dificultades para demostrar mediante la definición formal de la derivada la proposición la derivada de una función constante siempre es cero. Lo anterior muestra que más del $50 \%$ de los futuros profesores manifiesta carencias respecto al conocimiento del contenido especializado requerido para la solución de la tarea.

Para la Tarea 8 (Figura 2), se consideraron correctas aquellas respuestas en las que se obtuvo la velocidad pedida de la pelota a partir de procedimientos y justificaciones válidas. Las respuestas correctas están relacionadas con el tipo de configuración por aproximación bilateral (apartado 3.2.4). Las respuestas parcialmente correctas, relacionadas con la configuración por aproximación por la izquierda o derecha (apartado 3.2.3), fueron aquellas en las que se utilizaron procedimientos y justificaciones que no son del todo erróneos, pero tampoco son válidos para encontrar la velocidad pedida.

Como incorrectas se consideraron aquellas respuestas en las que no se halla la velocidad de la pelota para $t=0.4$, debido a que los procedimientos seguidos y las justificaciones dadas no eran válidos. Las respuestas incorrectas están relacionadas con los tipos de configuraciones descritas en los apartados 3.2.2 y 3.2.1. La Tabla 2 muestra los resultados obtenidos para el grado de corrección de la tarea 8.

Tabla 2 - Frecuencias y porcentajes para el grado de corrección de la Tarea 8

\begin{tabular}{|c|c|c|}
\hline \multirow{2}{*}{ Grado de Corrección } & \multicolumn{2}{|c|}{ Tarea 8} \\
\hline & Frecuencia & Porcentaje \\
\hline Correcta & 1 & 1,9 \\
\hline Parcialmente correcta & 5 & 9,4 \\
\hline Incorrecta & 23 & 43,4 \\
\hline No responden & 24 & 45,3 \\
\hline Total & 53 & 100 \\
\hline
\end{tabular}

Es posible observar a partir de los datos que se presentan en la Tabla 2 que tan sólo un estudiante para profesor $(1,9 \%)$ logra resolver correctamente la tarea, y cinco $(9,4 \%)$ dan una 
respuesta parcialmente correcta. Esto sugiere que, al menos, el $88,7 \%$ de los futuros profesores exhiben carencias respecto del conocimiento ampliado requerido para resolver la tarea.

La Tabla 3 presenta los resultados obtenidos para la Tarea 4, en relación con la variable cualitativa tipo de configuración cognitiva.

Tabla 3 - Frecuencias y porcentajes para el tipo de configuración cognitiva del ítem a) de la Tarea 4

\begin{tabular}{|c|c|c|}
\hline \multirow{2}{*}{ Tipo de configuración cognitiva } & \multicolumn{2}{|c|}{ Tarea 4: ítem a) } \\
\hline & Frecuencia & Porcentaje \\
\hline Analítica - extensiva & 15 & 28,3 \\
\hline Analítica - intensiva & 20 & 37,7 \\
\hline Trazado de tangentes & 2 & 3,8 \\
\hline Uso de situaciones particulares de variación & 1 & 1,9 \\
\hline Límite de las tasas medias de variación & 5 & 9,4 \\
\hline No dan solución & 10 & 18,9 \\
\hline Total & 53 & 100 \\
\hline
\end{tabular}

La Tabla 3, señala que el 69,8\% de los futuros profesores proporciona una solución (analíticas-extensivas, analíticas-intensivas, trazado de tangentes) en la que la derivada en un punto se interpreta como la pendiente de la recta tangente. El 11,3\% interpreta, en su solución, la derivada como razón instantánea de cambio. Este hecho, aunado a las evidencias que surgen a partir del análisis de los distintos tipos de configuración (apartado 3.1), sugieren una aparente desconexión entre los distintos significados de la derivada y diversas interpretaciones usadas por los estudiantes. Dicha desconexión se torna más evidente en las soluciones que los estudiantes presentan en la tarea 8. Así, debido a la relación existente entre la tarea y el tipo de conocimiento que evalúa, queda claro que los estudiantes presentan carencias no sólo en cuanto al conocimiento especializado (uso de distintas representaciones, uso de distintos significados de la derivada, resolución del problema mediante diversos procedimientos, dar diversas argumentaciones válidas para explicar dichos procedimientos etc.), sino también en relación con el conocimiento común requerido para resolver la tarea.

Finalmente, la Tabla 4 presenta los resultados obtenidos respecto a la variable tipo de configuración cognitiva para la tarea 8 (Figura 2). A partir de los datos obtenidos en el análisis realizado para cada tipo de configuración cognitiva (apartado 3.2) se puede observar, en la Tabla 4, que 17 (32\%) de los futuros profesores parecen no distinguir la diferencia entre función derivada y derivada en un punto. Las configuraciones cognitivas asociadas a la actividad matemática desarrollada por estos 17 profesores están contempladas en: mediante la relación física $\mathrm{v}=\mathrm{d} \mathfrak{d}$ ” " y aproximación por la izquierda o derecha. Este resultado, referente a la 
problemática para distinguir la diferencia entre la función derivada y la derivada en un punto ha sido reportado en otras investigaciones (INGLADA; FONT, 2003; BADILLO; AZCÁRATE; FONT, 2005; BADILLO; AZCÁRATE; FONT, 2011).

Tabla 4 - Frecuencias y porcentajes para el tipo de configuración cognitiva para la Tarea 8

Tipo de configuración cognitiva

\begin{tabular}{l}
\hline Patrón numérico \\
\hline Uso de la relación física $v=d / t$ \\
\hline Aproximación por la izquierda o derecha \\
\hline Aproximación bilateral \\
\hline No dan evidencia de su solución \\
\hline Total \\
\hline
\end{tabular}

\section{Tarea 8}

\begin{tabular}{cc}
\hline Frecuencia & Porcentaje \\
\hline 3 & 5,7 \\
\hline 13 & 24,5 \\
\hline 4 & 7,5 \\
\hline 2 & 3,8 \\
\hline 31 & 58,5 \\
\hline 53 & 100 \\
\hline
\end{tabular}

Esta desconexión aparente entre la interpretación de la derivada en un punto y la función derivada induce al error de responder la pregunta, ¿Cuál es la velocidad de la pelota en el instante $t=0.4 \mathrm{~s}$ ? mediante el cálculo de la velocidad promedio. Parece probado que los futuros profesores no relacionan los distintos significados para la derivada. Los estudiantes parecen ignorar que la relación $\mathrm{v}=\mathrm{d} / \mathrm{t}$ representa la velocidad promedio de la pelota correspondiente a dos tiempos distintos. Esta velocidad promedio se relaciona, a su vez, con la pendiente de alguna recta secante a la función desplazamiento, lo cual no se corresponde con una interpretación para la derivada.

\section{Reflexiones Finales}

Los resultados obtenidos a partir del análisis, cuantitativo y cualitativo, de las resoluciones que los estudiantes dieron a las tareas aquí analizadas, señalan que los futuros profesores exhiben ciertas dificultades para resolver tareas relacionadas con el conocimiento especializado y ampliado sobre la derivada. Las resoluciones dadas a la Tarea 4 muestran que se da mejor desempeño cuando se usa la derivada en un punto en su acepción como pendiente de la recta tangente. Además, en los resultados presentados en la Tabla 1 se observa que el $56,6 \%$ de los futuros profesores tuvieron problemas para demostrar, mediante la definición formal de la derivada la proposición la derivada de una función constante siempre es cero, lo cual sugiere que dichos estudiantes no dominan la práctica de la demostración cuando esta conlleva el uso de la derivada como límite de tasas medias de variación. 
Los resultados obtenidos en la Tarea 8, muestran las dificultades de los futuros profesores cuando tienen que usar la derivada como razón instantánea de cambio en el caso de una situación de cierta complejidad.

Se ha evidenciado cómo el conocimiento común del contenido no es suficiente para abordar tareas propias de la enseñanza, para las que se requiere no solo cierto nivel de conocimiento del contenido especializado (PINO-FAN; GODINO; FONT, 2013), sino también de conocimiento ampliado. Así mismo, se advierte una aparente desconexión entre los distintos significados de la derivada. Las respuestas de los futuros profesores muestran el complejo entramado de prácticas, objetos y procesos matemáticos puestos en juego en la resolución de las tareas relacionadas con la derivada. La toma de conciencia de esta complejidad es necesaria tanto para los formadores, para que den oportunidades a los profesores de desarrollar el conocimiento requerido para la enseñanza de la derivada, como para los propios futuros profesores, para que puedan desarrollar y evaluar la competencia matemática en sus futuros estudiantes.

Finalmente, debemos destacar que el análisis de las prácticas matemáticas, y configuraciones de objetos y procesos, se muestra como una herramienta potente para la identificación y caracterización de los conocimientos relativos a la faceta epistémica del CDM, en tanto que proporciona pautas y criterios para analizar dichos tipos de conocimientos manifestados por los futuros profesores. Las configuraciones cognitivas, descritas en la sección 3, permiten identificar los significados que los futuros profesores atribuyen a los objetos puestos en juego en las soluciones dadas a las tareas. Las categorías, o tipos de configuraciones cognitivas, en las cuales se han agrupado las soluciones que éstos dan a cada una de las tareas, se obtienen y describen a partir de las configuraciones de objetos y procesos evidenciadas en sus soluciones.

\section{Reconocimientos}

Trabajo realizado en el marco de los proyectos de investigación sobre formación de profesores, EDU2012-31869 (Universidad de Granada) y EDU2012-32644 (Universidad de Barcelona). 


\section{Referencias}

ARTIGUE, M.; BATANERO, C.; KENT, P. Mathematics thinking and learning at post-secondary level. In Lester F. K. (Ed.). Second Handbook of Research on Mathematics Teaching and Learning. Charlotte, N.C: NCTM and IAP, 2007. p. 1011-1049.

ASIALA, M.; COTTRILL, J.; DUBINSKY, E.; SCHWINGENDORF, K. The development of student's graphical understanding of the derivate. Journal of Mathematical Behavior, New Jersey, v. 16, n. 4, p. 399-431, Dec.1997.

BADILLO, E.; AZCÁRATE, C.; FONT, V. Conflictos semióticos relacionados con el uso de la notación incremental y diferencial en libros de física y de matemáticas del bachillerato. Enseñanzas de las Ciencias, Barcelona, Número extra, VII Congreso, p. 1-6, 2005.

BADILLO, E.; AZCÁRATE, C.; FONT, V. Análisis de los niveles de comprensión de los objetos $f^{\prime}(a)$ y $f^{\prime}(x)$ en profesores de matemáticas. Enseñanza de las Ciencias, Barcelona, v. 29, n. 2, p. 191-206, Jun. 2011.

BAKER, B.; COOLEY, L.; TRIGUEROS, M. A calculus graphing schema. Journal for Research in Mathematics Education, Reston, V.A., v. 31, n. 5, p. 557-578, Nov. 2000.

BALL, D.L. Bridging practices: Intertwining content and pedagogy in teaching and learning to teach. Journal of Teacher Education, Pennsylvania, v. 51, n. 3, p. 241-247, May. 2000.

ÇETIN, N. The ability of students to comprehend the function-derivative relationship with regard to problems from their real life. PRIMUS, England and Wales, v.19, n.3, p. 232-244, Apr. 2009.

FONT, V. Procediments per obtenir expressions simbòliques a partir de gràfiques. Aplicacions a la derivada [Procedures for obtaining symbolic expressions from graphs: Applications in relation to the derivative]. 1999. 782 p. Tesis (Doctorado en Filosofía y Ciencias de la Educación), Departament de Didàctica de les Ciències Experimentals i la Matemàtica, Universitat de Barcelona, España, 1999.

FONT, V.; GODINO, J.D.; GALLARDO, J. The emergence of objects from mathematical practices. Educational Studies in Mathematics, Berlin, v. 82, n. 1, p. 97-124, Jan. 2013.

GARCÍA, M.; LLINARES, S.; SÁNCHEZ-MATAMOROS, G. Characterizing thematized derivative schema by the underlying emergent structures. International Journal of Science and Mathematics Education, Taiwán, v. 9, n. 5, p. 1023-1045, Oct. 2011.

GAVILÁN, J.; GARCÍA, M.; LLINARES, S. Una perspectiva para el análisis de la práctica del profesor de matemáticas. Implicaciones metodológicas. Enseñanza de las ciencias, Barcelona, v. 25, n. 2, p. 157-170, Jun. 2007.

GODINO, J.D. Un enfoque ontológico y semiótico de la cognición matemática. Recherches en Didactique des Mathématiques, Grenoble, v. 22, n. 2/3, p. 237-284, Nov. 2002.

GODINO, J.D.; BATANERO, C.; FONT, V. The onto-semiotic approach to research in mathematics education. ZDM. The International Journal on Mathematics Education, Berlin, v. 39, n. 1, p. 127135, Mar. 2007.

GODINO, J.D. Categorías de análisis de los conocimientos del profesor de matemáticas. Unión, Revista Iberoamericana de Educación Matemática, Buenos Aires, v. 20, p. 13-31, Dic. 2009. 
GODINO, J.D. Indicadores de idoneidad didáctica de procesos de enseñanza y aprendizaje de las matemáticas. Cuadernos de Investigación y Formación en Educación Matemática, Costa Rica, Año 8, v. 11, p. 111-132, Dic. 2013.

GODINO, J.D.; FONT, V.; WILHELMI, M.; LURDUY, O. Why is the learning of elementary arithmetic concepts difficult? Semiotic tools for understanding the nature of mathematical objects. Educational Studies in Mathematics, Berlin, v. 77, n. 2-3, p. 247-265, Jul. 2011.

GODINO, J.D.; PINO-FAN, L. The mathematical knowledge for teaching. A view from onto-semiotic approach to mathematical knowledge and instruction. In: UBUZ, B.; HASER, Ç.; MARIOTTI, M. (Ed.). Proceedings of the Eighth Congress of the European Society for Research in Mathematics Education. Antalya, Turkey: CERME, 2013. p. 3325-3326.

HILL, H.C.; BALL, D.L.; SCHLLING, S.G. Unpacking pedagogical content knowledge of students. Journal for Research in Mathematics Education, Reston, V.A., v. 39, n. 4, 372-400, Jul. 2008.

INGLADA, N.; FONT, V. Significados institucionales y personales de la derivada. Conflictos semióticos relacionados con la notación incremental. En: Seminario Inter-Universitario de Investigación en Didáctica de la Matemática, 19, 2003, Córdoba. Actas de las XIX Jornadas del SIIDM, 2003, p. 1-18.

JOHNSON, R.B.; ONWUEGBUZIE, A. Mixed methods research: a research paradigm whose time has come. Educational Researcher, Washington, v. 33, n.7, p.14-26, Oct. 2004.

PINO-FAN, L. Evaluación de la faceta epistémica del conocimiento didáctico-matemático de futuros profesores de bachillerato sobre la derivada. 2013. 418 p. Tesis (Doctorado en Didáctica de la Matemática), - Facultad de Ciencias de la Educación, Universidad de Granada, Granada, España, 2013.

PINO-FAN, L.; FONT, V.; GODINO, J. D. Faceta epistémica del conocimiento didáctico-matemático sobre la derivada. Educação Matemática Pesquisa, São Paulo, Brasil, v.13, n.1, p. 141-178, Ene. 2011.

PINO-FAN, L.; FONT, V.; GODINO, J. D. El conocimiento didáctico-matemático de los profesores: pautas y criterios para su evaluación y desarrollo. En Dolores, C.; García, M.; Hernández, J.; Sosa, L. (Ed.). Matemática Educativa: La formación de profesores. México, D. F.: Ediciones D. D. S. y Universidad Autónoma de Guerrero, 2014, p. 137-151.

PINO-FAN, L.; GODINO, J.D.; FONT, V.; V. Diseño y aplicación de un instrumento para explorar la faceta epistémica del conocimiento didáctico-matemático de futuros profesores sobre la derivada ( $1^{\mathrm{a}}$ Parte). REVEMAT, Florianópolis, S. C., Brasil, v. 8, n. 2, p. 1-49, Dic. 2013.

ROWLAND, T.; HUCKSTEP, P.; THWAITES, A. Elementary teachers' mathematics subject knowledge: The knowledge quartet and the case of Naomi. Journal of Mathematics Teacher Education, Netherlands, v. 8, n. 3, p. 255-281, Jun. 2005.

SÁNCHEZ-MATAMOROS, G.; GARCÍA, G.; GARCÍA, M.; LLINARES, S. El desarrollo del esquema de derivada. Enseñanza de las Ciencias, Barcelona, v. 24, n. 1, p. 85-98, Mar. 2006.

SÁNCHEZ-MATAMOROS, G.; FERNÁNDEZ, C.; VALLS, J.; GARCÍA, M.; LLINARES, S. Cómo estudiantes para profesor interpretan el pensamiento matemático de los estudiantes de bachillerato. La derivada de una función en un punto. En Estepa, A.; Contreras, A.; Deulofeu, J.; Penalva, M.C.; 
García, F.J.; Ordoñez, L. (Ed.). Investigación en Educación Matemática XVI. Jaén: SEIEM, 2012, p. 497-508.

SCHOENFELD, A.; KILPATRICK, J. Towards a theory of profiency in teaching mathematics. In: Tirosh, D.; Wood, T. L. (Ed.). Tools and processes in mathematics teacher education. Rotterdam: Sense Publishers, 2008, p. 321-354.

SHULMAN, L.S. Those who understand: Knowledge growth in teaching. Educational Researcher, Washington, v. 15, n. 2, p. 4-14, Feb. 1986.

VIHOLAINEN, A. Finnish mathematics teacher student's informal and formal arguing skills in the case of derivative. Nordic Studies in Mathematics Education, Göteborg, v. 13, n. 2, p. 71-92, Jun. 2008. 